\title{
Closed-form solution for minimizing power consumption in coordinated transmissions
}

Qimei Cui ${ }^{*}$, Bing Luo ${ }^{1}$, Xueqing Huang ${ }^{1}$, Alexis Dowhuszko ${ }^{2}$ and Jun Jiang ${ }^{3}$

\begin{abstract}
The growth in the demand of energy, and its consequent contribution to the greenhouse effect, gives rise to new challenges in the design of future wireless networks. Keeping in mind these requirements, in this article we study the power allocation problem in the downlink of an orthogonal frequency division multiple access (OFDMA) system, where two (or more) coordinated transmission points (CTPs) should find the best way to allocate their transmit power through the multiple orthogonal sub-channels of the system. The ultimate goal of the power allocation scheme is to minimize the joint power consumption of the system, but verifying at the same time the target throughput and the individual power constraint per CTP. The power allocation problem is formulated as a constrained optimization problem, and a group of closed-form power allocation solutions are derived. Based on the derived solutions (that take the form of the traditional water-filling but demanding cooperation among CTPs), a novel power allocation algorithm with joint minimization power consumption (JMPC-PA) is proposed. Numerical results are presented to verify the optimality of the results that were obtained by the JMPC-PA scheme. It is important to note that, due to the flexibility that exist in the definition of CTPs in this article, the derived power allocation scheme is valid for any kind of network that incorporates the coordinated multipoint transmission feature in its design.
\end{abstract}

Keywords: power Allocation, coordinated multi-point transmission (CoMP), orthogonal frequency division multiplexing access (OFDMA), water-filling (WF)

\section{Introduction}

Orthogonal frequency division multiplexing access (OFDMA) is a promising technique for high data rate transmission in wideband wireless systems [1,2]. In OFDMA systems, the total bandwidth is divided into many orthogonal narrowband subcarriers, with different subcarriers allocated to different users to enable flexible multi-user access. Transmission power allocation represents an effective way to increase the throughput of wireless communication systems [3]. According to different optimization objectives and constraints, adaptive power allocation schemes for OFDMA systems can be roughly divided into two categories: rate adaptive (RA) schemes and margin adaptive (MA) schemes. Rate adaptive schemes seek the maximization of the system throughput under a total and/or individual transmit

\footnotetext{
* Correspondence: cuiqimei@bupt.edu.cn

${ }^{1}$ Key Laboratory of Universal Wireless Communications, Ministry of Education, Beijing University of Posts and Telecommunications, Beijing, China Full list of author information is available at the end of the article
}

power constraints [4-6], while MA schemes try to minimize the overall transmission power with constraints on individual and/or system data rates [7-9]. It is shown that the optimal power allocation policy of the above schemes often leads to the form of a WF solution. However, traditional WF solutions are simple to evaluate because all of them consider a single power constraint (known as waterlevel). As a consequence, it is quite straightforward to compute the solution numerically. However, some other optimization problems (such as multi-user or multiple transmission points) would result in complicated non-convex problems. For such cases, it may be difficult to obtain closed-form solutions or practical algorithms.

Recently, the concept of cooperative communications has been proposed for wireless networks, such as macrocellular networks and wireless ad-hoc networks [10-13]. The basic idea behind cooperative communications is to allow the nodes in the wireless networks to transmit their information signals coordinately, to 
improve the quality of the communication (via spatial diversity) or to increase the achievable data rates (through spatial multiplexing). In both cases, the ultimate goal is to boost the system performance and achieve a better usage of the system resources. One typical technology in this context is the so-called coordinated multi-point transmission/reception (CoMP), which has been considered as an effective tool to improve the coverage of high data rates and the celledge throughput for 3GPP long term evolution (LTE) advanced [14].

Motivated by the coordinated feature of CoMP, a new cooperative power allocation scheme was presented in [15], where two coordinated transmission points (CTPs) allocated jointly their constrained transmit power to the multiple orthogonal subchannels based on their channel state information (CSI) that they exchanged. In order to maximize the system throughput, a closed-form solution known as joint-waterfilling (Jo-WF) solution was obtained by Luo et al. [15], when solving the constrained non-convex optimization problem that arose in that situation. The solution turned out to take the form of tradition WF, and also had a cooperative feature. In green radio framework, however, the myopic maximization of system throughput does not make much sense. On the contrary, it is more important to minimize the total cost of transmission (i.e., the transmission power), while setting the constraints in the individual transmit power of each CTP power to attain a given target throughput.

In this article, we extend our previous study and analyze the dual power allocation problem in an OFDMA system. We present an effective way to coordinate the transmission between adjacent CTPs, seeking the minimization of the total transmit power while meeting the throughput requirements. To simplify the analysis we assume that the subchannels that are available to serve a given user are selected beforehand, e.g., using an arbitrary scheduling algorithm. This simplification allows us to put the emphasis in the design of the power allocation scheme, focusing our attention in the formulation of our objective task into a non-convex optimization problem. A group of closed-form solutions are obtained. Based on the derived solutions, a novel power allocation algorithm with joint minimization power consumption (JMPC-PA) is proposed. Numerical simulation results allow to show that, when compared to equal power allocation (EPA) scheme, the proposed JMPC-PA scheme provides a significant gain in terms of total power consumption.

Motivated by the regular theoretical derivation that was obtained in the two-CTP case, we also extend the derived results into an arbitrary K-CTP case, an scenario that can be considered more significant for practical networks. Due to the definition of CTP in this study is very flexible (i.e., it could represent both, a base station or a relay node), it is important to highlight that the JMPC-PA scheme can be applied to any kind of cooperative networks, such as next-generation macrocellular networks, heterogeneous networks, and ad-hoc networks.

The rest of the article is organized as follows. Section 2 presents the coordinated transmission model. Section 3 introduces the closed-form power allocation solutions for the 2-CTP case, while Section 4 extends the analysis to the more generic K-CTP (i.e., with an arbitrary large number of coordinated sources). Simulation results are presented in Section 5. Finally, the concluding remarks of the paper are summarized in Section 6 .

\section{Coordinated transmission model}

We consider a downlink multi-user OFDM system, in which two CTPs coordinately transmit their power to the coordinative zone users as depicted in Figure 1. In Figure 1, the CTPs are denoted by the solid squares and the users are denoted by the circles. Each CTP has all the information intended to the users in the coordinative zone via a wireline connection. In order to simplify the mathematical derivation, we assume that the two CTPs have the same power constraint $P_{0}$, and that they share the same overall bandwidth $B$, which is divided into $N$ orthogonal narrow-band subcarriers. Each user feeds back the CSI to its corresponding CTP via a feedback channel, and the instantaneous CSI can be exchanged reliably and fast between the two CTPs.

In order to focus solely on power allocation, we do not explicitly consider subcarrier scheduling here. However, it is noted that the power allocation results presented in this article are valid for any scheduling strategy. ${ }^{a}$ Besides, in this article we only focus on the total system capacity, leaving aside fairness issues concerning the way in which the common resources are shared among users. We also emphasize that our analysis is valid for any kind of coordinated transmission network, as long as the sum of orthogonal subcarrier capacities is a relevant performance metric.

In a multi-carrier system, the sum of the individual capacities per carrier represents the total system capacity. We suppose that a certain group of $\mathrm{N}$-subcarriers has been selected for coordinated transmission by an arbitrary scheduling algorithm. Let us assume that the subcarriers are narrow enough to undergo flat fading, and that the channel gains are constant within a given transmission time interval. Thus, the achievable (sum rate) throughout given by the Shannon formula attains the form 


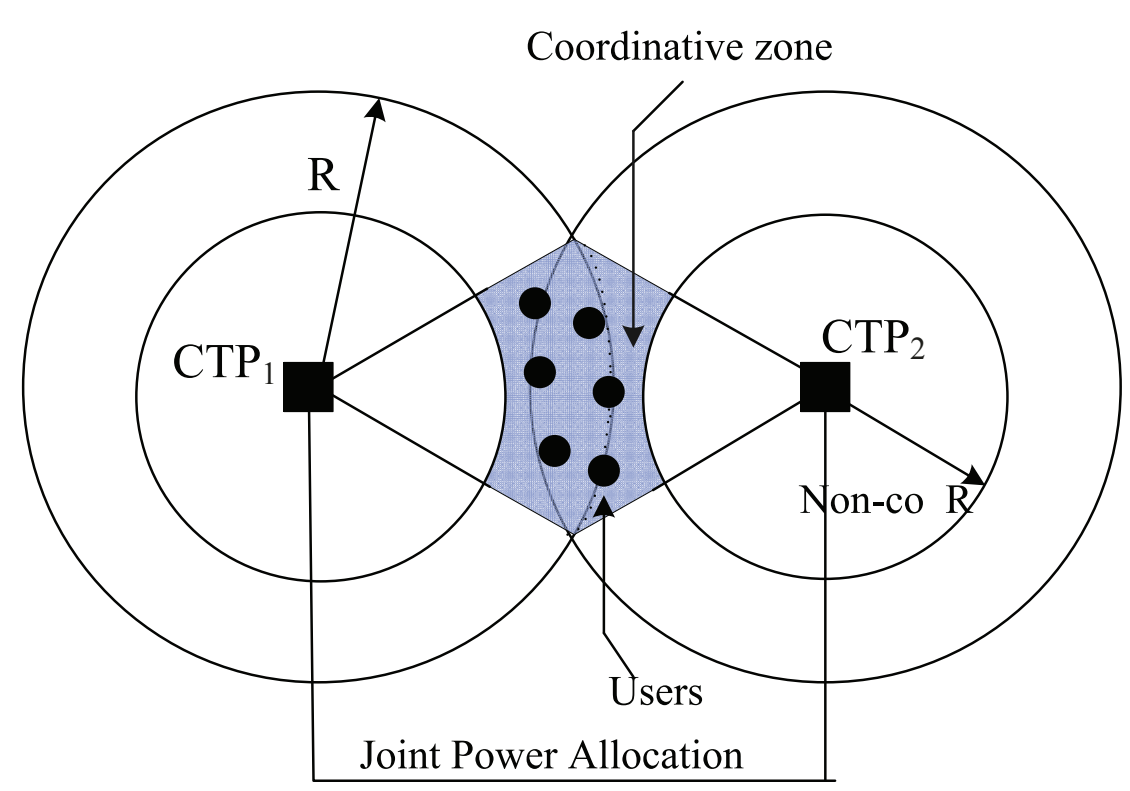

Figure 1 System model with two coordinated transmission points (CTP).

$$
R=\sum_{j=1}^{N} R_{j}=\sum_{j=1}^{N} \frac{B}{N} \log _{2}\left(1+\frac{\sum_{i=1}^{2} P_{i, j}\left|H_{i, j}\right|^{2}}{\sigma_{j}^{2}}\right),
$$

where $\sigma_{j}^{2}=N_{0} B / N$ represents the power of additive white Gaussian noise at the $j$ th subcarrier, $P_{i j}$ denotes the allocated transmission power from CTP $i$ to the $j$ th subcarrier, and $H_{i, j}$ represents the corresponding channel gain between the CTP $i$ and the $j$ th subcarrier.

The aim of this article is to minimize the joint transmit power for both CTPs, while satisfying at the same time the system throughput requirement $R_{S}$ as well as the individual transmit power constraint per CTP. In mathematical terms, this is equivalent to solve the following optimization problem:

$$
\begin{aligned}
& \text { minimize } P_{\text {overall }}=\sum_{j=1}^{N} P_{1, j}+\sum_{j=1}^{N} P_{2, j} \\
& \text { subject to } R=R_{S}, \sum_{j=1}^{N} P_{i, j} \leq P_{0}, \quad i=1,2 .
\end{aligned}
$$

\section{Closed-form power allocation solutions}

We search for the optimal coordinated power allocation by approaching the following optimization problem:

$$
\mathbf{P}_{i}^{*}=\left(P_{i, 1}^{*}, \ldots, P_{i, N}^{*}\right)=\arg \min _{\mathbf{P}_{i} \in \Omega^{N}} P_{\text {overall },} \quad i=1,2,
$$

where

$$
\Omega^{N}=\left\{\mathbf{P}_{i}\left|\forall j \in\{1, \ldots, N\}, 0 \leq P_{i, j} \leq P_{0}, \sum_{\mathbf{j}=1}^{\mathrm{N}} P_{i, j} \leq P_{0}\right|\right\}
$$

is the feasible set. Since $\Omega^{N}$ is a closed and bounded set, and $R: \Omega^{N} \rightarrow \mathbb{R}$ is continuous, function (1) has a solution [[16], Theorem 0.3].

For the sake of mathematical derivation, we denote $\gamma_{i, j}=\left(P_{0}\left|H_{i, j}\right|^{2}\right) / \sigma_{j}^{2}$ and $x_{i, j}=P_{i, j} / P_{0}$. It is indicated that $\gamma_{i, j}$ is the SNR associated with CTP $i$ over the $j$ th subchannel when assuming the entire power $P_{0}$ is allocated to the $j$ th subchannel, and $x_{i, j}$ represents the power allocation ratio. Since the logarithm is monotonically increasing function, the objective (2) combined with the constraints can be described as

$$
\begin{aligned}
\text { minimize } & Z=P_{\text {overall }} / P_{0}=\sum_{j=1}^{N} x_{1, j}+\sum_{j=1}^{N} x_{2, j} \\
\text { subject to } & \prod_{j=1}^{N}\left(1+\sum_{i=1}^{2} \gamma_{i, j} x_{i, j}\right)=2^{R_{S} /\left(\frac{B}{N}\right)}=2^{R_{S}^{*}}, \\
& \sum_{j=1}^{N} x_{i, j} \leq 1, \quad i=1,2,
\end{aligned}
$$

where $Z \leq 2$ represents the total power consumption ratio.

In order to obtain a closed-form expression for the required power allocation, we divide the constrained problem into two different cases: 
a) The power constraint is assumed to be large enough, so that solving the primal objective is equivalent to solving an unconstrained problem. In this case, the power constraint at each CTP (i.e., $\sum_{j=1}^{N} x_{i, j} \leq 1$ for $i=1,2)$ is always satisfied.

b) The power constraint is not large enough, so that one of the CTPs may exceed the power constraint if the distribution of the users is not even. In this situation, the solution in (a) leads to $\sum_{j=1}^{N} x_{1, j}>1$ or $\sum_{j=1}^{N} x_{2, j}>1$. In this situation the exceeded CTP should provide its full power for transmission, while the other CTP should increase its transmit power until it meets the throughput requirement for the system. In mathematic terms, the constraint in the primal objective should be changes to $\sum_{j=1}^{N} x_{1, j}=1$ and $\sum_{j=1}^{N} x_{2, j} \leq 1$, or to $\sum_{j=1}^{N} x_{2, j}=1$ and $\sum_{j=1}^{N} x_{1, j} \leq 1$. Actually, this case is more meaningful in practical systems.

\subsection{Optimal solution for the unconstrained case}

It is noted that for arbitrary $\gamma_{i, j}$, the likelihood of having $\gamma_{1, j}=\gamma_{2, j}$ for $j \in\{1,2\}$ in an actual system is almost zero. Without loss of generality, we divide the $N$ subchannels into two parts: the first part contains $M$ subchannels that satisfy $\gamma_{1, m}>\gamma_{2, m}$ for $m \in\{1,2, \ldots, M\}$, while the second part contains $K$ subchannels that satisfy $\gamma_{1, k}<\gamma_{2, k}$ for $k \in\{1,2, \ldots, K\}$. According to this model, $N=M+K$.

We first present the following lemma:

Lemma 1: When ignoring the power constraint $\sum_{j=1}^{N} x_{1, j} \leq 1 ; \sum_{j=1}^{N} x_{2, j} \leq 1$, the objective problem (4) can be degenerated into the unconstrained function

$$
\begin{array}{ll}
\text { minimize } & Z=\sum_{m=1}^{M} x_{1, m}+\sum_{k=1}^{K} x_{2, k} \\
\text { subject to } & \prod_{m=1}^{M}\left(1+\gamma_{1, m} x_{1, m}\right) \cdot \prod_{k=1}^{K}\left(1+\gamma_{2, k} x_{2, k}\right)=2^{R_{S}^{*},} \\
& M+K=N .
\end{array}
$$

Proof: To prove this lemma we use reductio ad absurdum. Assume that vectors $\mathbf{P}_{1}{ }^{*}=\left(x_{1, m}{ }^{*}, \ldots, x_{1, k}{ }^{*} \neq 0, \ldots\right)$ and $\mathbf{P}_{2}{ }^{*}=\left(x_{2, m}{ }^{*} \neq 0, \ldots, x_{2, k}{ }^{*}, \ldots\right)$ are optimal solutions for $m \in\{1, \ldots, M\}$ and $k \in\{1, \ldots, K\}$, which satisfy the throughput requirement and achieve the minimum $Z^{*}$. However, we could find another set of vectors $\mathbf{P}_{1}{ }^{* *}=\left(x^{* *}{ }_{1, m}=x^{*}{ }_{1, m}+\left(\gamma_{2, m} / \gamma_{1, m}\right) x^{*}{ }_{2, m}, \ldots, x_{1, k}{ }^{* *}=0 \ldots\right)$ and $\mathbf{P}_{2}{ }^{* *}=\left(x_{2, m}{ }^{* *}=0, \ldots, x^{* *}{ }_{2, k}=x^{*}{ }_{2, k}+\left(\gamma_{1, k} / \gamma_{2, k}\right) x_{1, k}^{*}, \ldots\right)$ which also satisfy the throughput requirement, and achieve a smaller $Z^{* * *}$, due to $\gamma_{1, m}>\gamma_{2, m}, \gamma_{1, k}<\gamma_{2, k}$ and

$$
\begin{aligned}
Z^{* *} & =\sum_{m=1}^{M}\left(x^{*}{ }_{1, m}+\frac{\gamma_{2, m}}{\gamma_{1, m}} x^{*}{ }_{2, m}\right)+\sum_{k=1}^{K}\left(x^{*}{ }_{2, k}+\frac{\gamma_{1, k}}{\gamma_{2, k}} x_{1, k}\right) \\
& <Z^{*}=\sum_{m=1}^{M}\left(x^{*}{ }_{1, m}+x^{*}{ }_{2, m}\right)+\sum_{k=1}^{K}\left(x_{2, k}^{*}+x^{*}{ }_{1, k}\right) .
\end{aligned}
$$

Therefore, the objective (4) could be equivalently transformed into (5). The degeneration implies that a group of $M$ subchannels (that verify $\gamma_{1, m}>\gamma_{2, m}$ ) should receive power allocation from CTP 1 , while the other $K=N-M$ subchannels (that verify $\gamma_{1, k}<\gamma_{2, k}$ ) should receive power allocation from CTP 2 . In other words, to achieve the goal of minimizing the total transmit power in the system, each CTP should select the better subchannels (according to the channel quality) to allocate power on them.

The degraded objective function (5) is strictly convex. Then, by Lagrange dual function we have that

$$
Z=\sum_{m=1}^{M} x_{1, m}+\sum_{k=1}^{K} x_{2, k}-\lambda\left[\prod_{m=1}^{M}\left(1+\gamma_{1, m} x_{1, m}\right) \cdot \prod_{k=1}^{K}\left(1+\gamma_{2, k} x_{2, k}\right)-2^{R_{s}^{k}}\right] .
$$

First, we derive $\lambda$ as

$$
\lambda=\frac{1}{2^{R_{S}^{*}}}\left(\frac{2^{R_{S}^{*}}}{\prod_{m=1}^{M} \gamma_{1, m} \cdot \prod_{k=1}^{K} \gamma_{2, k}}\right)^{\frac{1}{N}} .
$$

Then, the closed-form solution for the power allocation ratio at each CTP is obtained as follows:

$$
x_{1, m}=\lambda 2^{R_{s}^{*}}-\frac{1}{\gamma_{1, m}} \quad m \in\{1, \ldots, M\}, \quad x_{2, k}=\lambda 2^{R_{s}^{*}}-\frac{1}{\gamma_{2, k}} \quad k \in\{1, \ldots, K\} .
$$

Finally, the minimum total power consumption ratio is given by the following expression:

$$
Z=N\left(\frac{2^{R_{S}^{*}}}{\prod_{m=1}^{M} \gamma_{1, m} \cdot \prod_{k=1}^{K} \gamma_{2, k}}\right)^{\frac{1}{N}}-\sum_{m=1}^{M} \frac{1}{\gamma_{1, m}}-\sum_{k=1}^{K} \frac{1}{\gamma_{2, k}} .
$$

Note: As mentioned before, formula (9) only represents the optimal solution for the primal objective when the power constraint $\sum_{j=1}^{N} x_{i, j} \leq 1$ for $i=1,2$ holds. However, when the power constraint is not large enough and the user density is not evenly distributed, one of the CTPs may exceed its individual power constraint. In this situation, the solution in (9) leads to $\sum_{j=1}^{N} x_{1, j}>1$ or $\sum_{j=1}^{N} x_{2, j}>1$. Therefore, the exceeded CTP should provide its full power for transmission, and the power allocation for the other user should be 
recalculated to meet the throughput requirement for the system. The analyisis for this case is specified in the following section.

\subsection{Optimal solution for the constrained case}

Without loss of generality, we assume that CTP 1 is the overloaded transmission point. Thus, it is straightforward to know that CTP 1 should provide its full power for transmission (i.e., $\sum_{j=1}^{N} x_{1, j}=1$ ). Then the objective

(4) can be redefined as

$$
\begin{aligned}
\text { minimize } & Z=1+\sum_{j=1}^{N} x_{2, j} \\
\text { subject to } & \prod_{j=1}^{N}\left(1+\sum_{i=1}^{2} \gamma_{i, j} x_{i, j}\right)=2^{R_{S}^{*}} ; \\
& \sum_{j=1}^{N} x_{1, j}=1, \sum_{j=1}^{N} x_{2, j} \leq 1 .
\end{aligned}
$$

Before going forward in the analysis, we first need to present the following lemma.

Lemma 2: In order to minimize the overall power consumption, the optimal power allocation strategy consists in transmitting simultaneously from both CTPs in only one of the $\mathrm{N}$-subchannels. The rest of the subchannels should be divided in two groups, one per CTP. The selection of the subchannels for each group should be carried out according to the individual subchannel gains from each CTP.

Proof: By Lagrange dual function we have that

$$
\begin{aligned}
\Gamma= & 1+x_{2,1}+\cdots+x_{2, N}-\lambda\left\{\left(1+\gamma_{1,1} x_{1,1}+\gamma_{2,1} x_{2,1}\right) \cdots\right. \\
& {\left.\left[1+\gamma_{1, N}\left(1-x_{1,1}-\cdots-x_{1, N-1}\right)+\gamma_{2, N} x_{2, N}\right]-2^{R_{S}^{*}}\right\} . }
\end{aligned}
$$

For the sake of mathematic simplification, we denote $A_{j}=\left(1+\gamma_{1, j} x_{1, j}+\gamma_{2, j} x_{2, j}\right)$ and obtain the Hessian matrix $\nabla^{2} f$ as follows:

$$
\nabla^{2} \Gamma=\left[\begin{array}{cccc}
\frac{\partial^{2} \Gamma}{\partial x_{1,1}^{2}} & \frac{\partial^{2} \Gamma}{\partial x_{1,1} \partial x_{2,1}} & \cdots & \frac{\partial^{2} \Gamma}{\partial x_{1,1} \partial x_{2, N}} \\
\frac{\partial^{2} \Gamma}{\partial x_{2,1} \partial x_{1,1}} & \frac{\partial^{2} \Gamma}{\partial x_{2,1}{ }^{2}} & \cdots & \\
\vdots & & \ddots & \\
\frac{\partial^{2} \Gamma}{\partial x_{2, N} \partial x_{1,1}} & & \cdots & \frac{\partial^{2} \Gamma}{\partial x_{2, N^{2}}}
\end{array}\right] .
$$

The first-order principal minor determinant of $\nabla^{2} \Gamma$ is given by $\partial^{2} \Gamma / \partial x_{1,1}{ }^{2}=-2 \gamma_{1,1} \gamma_{1, N} A_{2} A_{3} \ldots A_{N-1}<0$, while the second-order principal minor determinant attains the form

$$
\frac{\partial^{2} \Gamma}{\partial x_{1,1}{ }^{2}} \cdot \frac{\partial^{2} \Gamma}{\partial x_{2,1}{ }^{2}}-\frac{\partial^{2} \Gamma}{\partial x_{1,1} \partial x_{2,1}} \cdot \frac{\partial^{2} \Gamma}{\partial x_{2,1} \partial x_{1,1}}=-\left(\lambda \gamma_{2,1} \gamma_{1, N} A_{2} A_{3} \ldots A_{N-1}\right)^{2}<0 .
$$

Therefore, the Hessian $\nabla^{2} \Gamma$ is indefinite since both, the first-order and the second-order principal minor determinant are negative. This means that function (12) has no extreme points, and as a consequence, the minimum value must be achieved on the domain boundary [17]. In other words, one of the $x_{i, 1}$ for $i \in\{1,2\}$ equals to 0 ; i.e., $A_{1}=\left(1+\gamma_{1,1} x_{1,1}+\gamma_{2,1} x_{2,1}\right)$ should be degenerated to $A_{1}^{\Delta}=\left(1+\gamma_{i, 1} x_{i, 1}\right)$ for $i \in\{1,2\}$.

Similarly, for arbitrary $A_{j}=\left(1+\gamma_{1, j} x_{1, j}+\gamma_{2, j} x_{2, j}\right)$, we still have the following equation

$$
\frac{\partial^{2} \Gamma}{\partial x_{1, j}{ }^{2}} \frac{\partial^{2} \Gamma}{\partial x_{2, j}^{2}}-\frac{\partial^{2} \Gamma}{\partial x_{1, j} \partial x_{2, j}} \frac{\partial^{2} \Gamma}{\partial x_{2, j} \partial x_{1, j}}=-\left(\lambda \gamma_{2, j} \gamma_{1, N} A_{1} \ldots A_{j-1} A_{j+1} \ldots A_{N-1}\right)^{2}<0 .
$$

Hence, in order to achieve the minimum, all the $A_{j}=$ $\left(1+\gamma_{1 j} x_{1 j}+\gamma_{2 j} x_{2 j}\right), j \in\{1, \ldots, N-1\}$ ought to be degenerated to $A_{j}^{\Delta}=\left(1+\gamma_{i, j} x_{i, j}\right)$ for $i \in\{1,2\}$. At the end, due to the constraint $x_{1, n}=1-\sum_{j=1, j \neq n}^{N} x_{1, j}$, a certain $A_{n}$ for $\mathrm{n} \in$ $\{1 \ldots \mathrm{N}\}$ is left with two additive variables $\left(1+\gamma_{1, n} x_{1, n}+\right.$ $\left.\gamma_{2, n} x_{2, n}\right)$, and the other $A_{j}$ for $j \in\{1 \ldots N\}(j \neq n)$ are degenerated to the form of $A_{j}^{\Delta}=\left(1+\gamma_{i, j} x_{i, j}\right)$ for $i=1$ or 2 . Thus, function (12) has been degenerated into strictly convex.

Based on the above analysis, the optimal power allocation mode (as depicted in Figure 2) is that only one of the $N$-subchannels (i.e., a certain $A_{n}$ ) should be transmitted together by the two CTPs; the others, according to the different subchannel gains, should be separated into two parts (i.e., $x_{1, j}$ or $x_{2, j}$ ) and transmitted by an individual CTP.

A specific 2-subchannel overloaded case is presented here to illustrate Lemma 2 by numerical simulation (see Figure 3). As an example of function (11), we assume $2^{R_{S}^{*}}=10$. Considering the power constraint, path loss attenuation, shadowing, and noise, we randomly generate $\gamma_{i, j}$ as follows: $\gamma_{1,1}=3.205, \gamma_{2,1}=2.311, \gamma_{1,2}=4.108, \gamma_{2,2}=$ 3.406. Figure 3 shows the minimum power consumption $Z$ versus ergodic power allocation ratio. Based on this figure it is possible to see that, the total power consumption reaches its minimum value (i.e., 1.229$)$ when $\left(x_{1,2}=0.44\right.$, $\left.x_{2,1}=0\right)$ and $\left(x_{1,1}=0.56, x_{2,2}=0.229\right)$. Note that this set of values verifies Lemma 2, that stated that the optimal power allocation is obtained on the domain boundary, when only one of the available subchannels is being used simultaneously by the two CTPs.

Now we are ready to solve the degraded convex problem, and obtain a closed-form solution for optimal power allocation. Without loss of generality, we consider that the $n$th subchannel represents the common channel which is used simultaneously by both CTPs. Then, we divide the $N-1$ subchannels into two groups: the first group of $M$ subchannels is used exclusively by CTP 1 , 


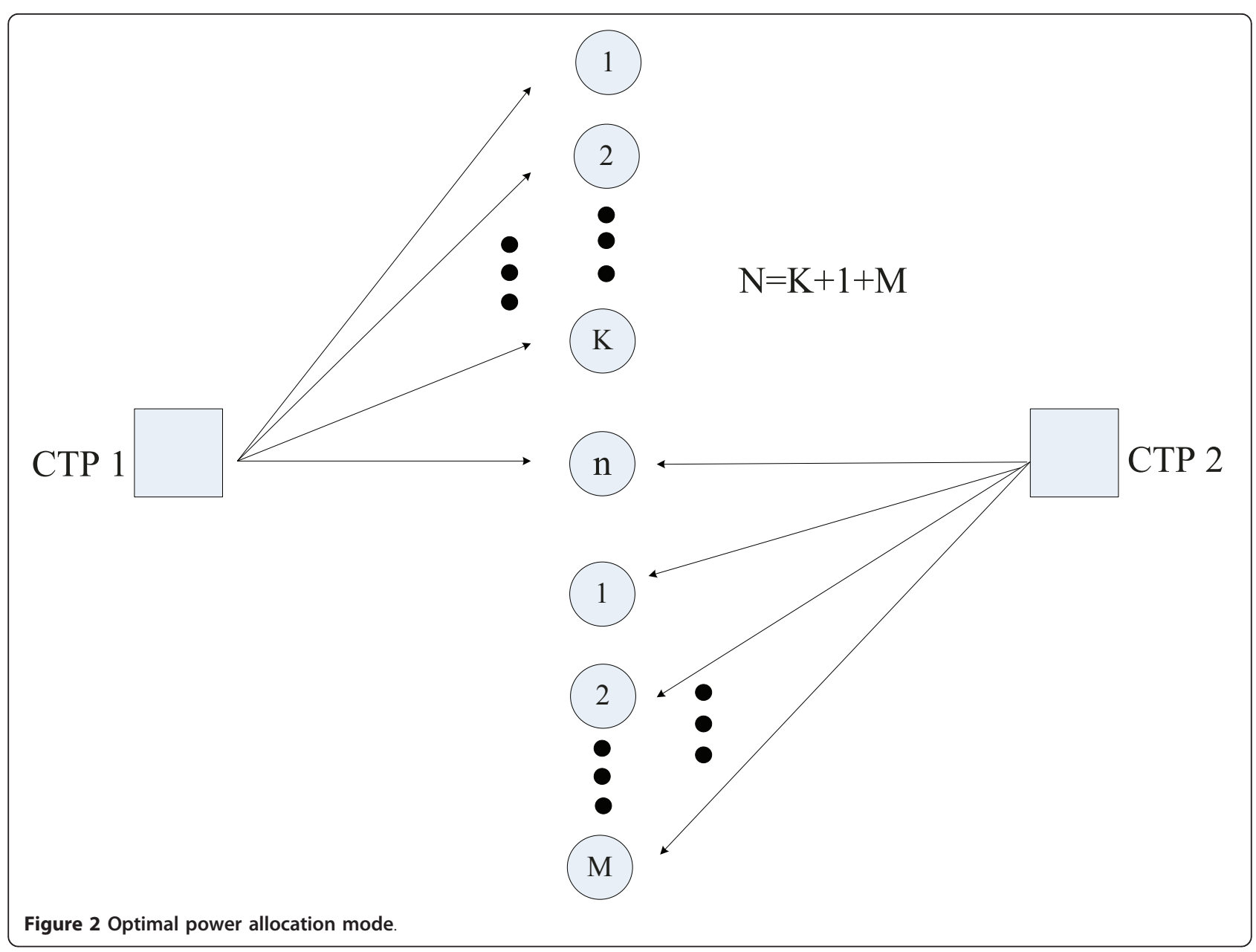

and the second group of $K=N-M-1$ subchannels is used solely by CTP 2 . Thus, the objective function (12) can be expressed as

$$
\begin{aligned}
& \Gamma=1+x_{2, n}+x_{2,1}+\ldots x_{2, K}-\lambda\left\{\prod_{m=1}^{M}\left(1+\gamma_{1, m} x_{1, m}\right) \cdot \prod_{k=1}^{K}\left(1+\gamma_{2, k} x_{2, k}\right)\right. \\
& \cdot\left[1+\gamma_{1, n}\left(1-\sum_{m=1}^{M} x_{1, m}\right) x_{1, n}+\gamma_{2, n} x_{2, n}\right]-2^{\left.R_{s}^{s}\right\}} .
\end{aligned}
$$

By partial derivation on (16), it is possible to see that

$$
\lambda=\frac{1}{2^{R_{S}^{*}}}\left(\frac{\left(\gamma_{1, n} / \gamma_{2, n}\right)^{M} 2^{R_{S}^{*}}}{\gamma_{2, n} \cdot \prod_{m=1}^{M} \gamma_{1, m} \cdot \prod_{k=1}^{K} \gamma_{1, k}}\right)^{\frac{1}{N}} .
$$

Then, the optimal solution is obtained as follows:

$$
\left\{\begin{array}{l}
x_{1, m}=\frac{\gamma_{2, n}}{\gamma_{1, n}} \lambda 2^{R_{S}^{*}}-\frac{1}{\gamma_{1, m}}, \quad x_{1, n}=1-\sum_{m=1}^{M} x_{1, m} \\
x_{2, k}=\lambda 2^{R_{S}^{*}}-\frac{1}{\gamma_{2, k}}, \quad x_{2, n}=\lambda 2^{R_{S}^{*}}-\frac{1+\gamma_{1, n} x_{1, n}}{\gamma_{2, n}} .
\end{array}\right.
$$

Remark: It is seen that the solutions (9) and (18) turn out to take the form of traditional WF solution, but having a coordinated feature. The coordinated feature here means that the optimal power allocation ratio $x_{1, m}$ correlates not only with $\gamma_{1, m}$ for $m \in(1, \ldots, M)$ and $\gamma_{2, k}$ for $k \in$ $(1, \ldots, K)$, but also depends on the relationship $\gamma_{2, n} / \gamma_{1, n}$ in (18). The optimal solution can be only obtained through perfect coordination between both CTPs. According to the derived solution, both CTPs have to determine jointly the power ratio that they should allocate on each of their active subchannel, to achieve an effective minimization of the overall power consumption of the system.

Finally, by $\gamma_{i, j}=\left(P_{0}\left|H_{i, j}\right|^{2}\right) / N_{0} \Delta B$ and $x_{i, j}=P_{i j} / P_{0}$, the optimal power allocation is given by

$$
\begin{aligned}
& \mathbf{P}_{1}^{*}=(P_{1,1}{ }^{*}, \ldots, P_{1, M}{ }^{*}, P_{1, n} *, \underbrace{0, \ldots, 0}_{K}), \\
& \mathbf{P}_{2}^{*}=(\underbrace{0, \ldots, 0}_{M}, P_{2, n}{ }^{*}, P_{2,1}{ }^{*}, \ldots, P_{2, N-M-1}{ }^{*}), \\
& M=\underset{M \in\{1, \ldots, N-1\}}{\arg \min P_{\text {overall }} .}
\end{aligned}
$$




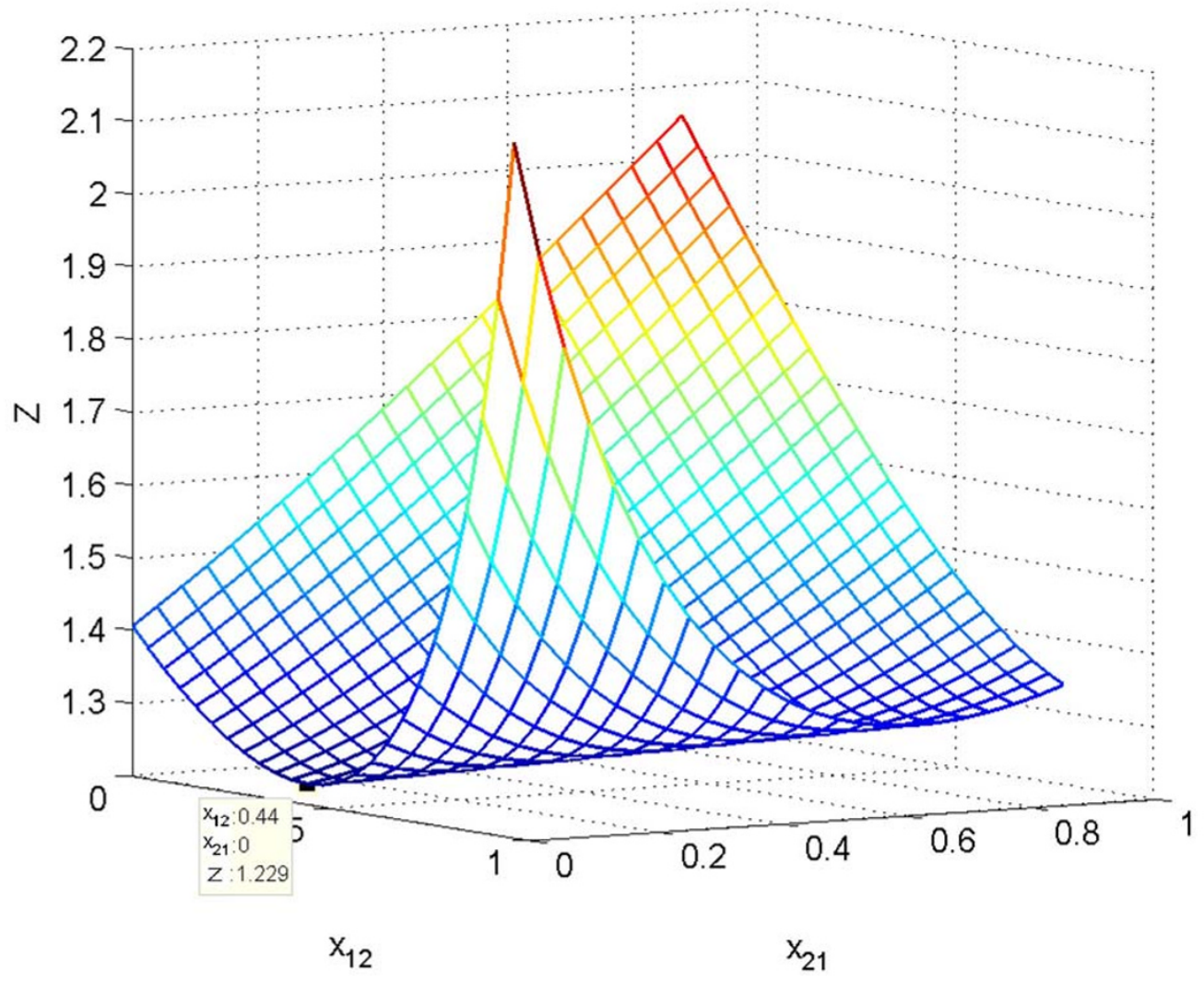

Figure 3 Minimum power consumption $Z$ versus ergodic power allocation ratio $x_{12}$ and $x_{21}$.

The intuition behind the optimal power allocation in (9) and (18) is also to take advantage of good channel conditions: when subchannel condition is good, more power (and a higher data) rate is sent over the subchannel. On the other hand, as the subchannel quality degrades, less power (and a lower data rate) is sent over the channel. It is emphasized that the solution should be satisfied by $P_{i, j} \geq 0$; i.e., if the channel SNR falls below the cutoff threshold, the channel is not used and

$$
P_{i, j}{ }^{*}=\left[P_{i, j}\right]^{+}=\max \left\{0, P_{i, j}\right\}
$$

results. Hence, an iterative JMPC-PA algorithm is presented (as depicted in Figure 4), not only to determine the optimal power allocation vectors $\mathbf{P}_{1}{ }^{*}$ and $\mathbf{P}_{2}{ }^{*}$, but also to evaluate the solutions numerically.

\section{Optimal solution for the arbitrary K-CTP case}

This section extends the previous analysis and lemmas from the simple 2-CTP case to the more general K-CTP situation. It is important to note that the assumptions in this section are similar to the ones that have been already presented in the previous section.
The general form for the objective function (2) in presense of $K$ CTPs attains the form

$$
\begin{array}{ll}
\text { minimize } & Z=\sum_{j=1}^{N} x_{1, j}+\sum_{j=1}^{N} x_{2, j}+\ldots \sum_{j=1}^{N} x_{K, j} \\
\text { subject to } & \left(1+\gamma_{1,1} x_{1,1} \ldots+\gamma_{K, 1} x_{K, 1}\right) \ldots\left(1+\gamma_{1, N} x_{1, N} \ldots+\gamma_{K, N} x_{K, N}\right)=2^{R_{S}^{*}}
\end{array}
$$

Regularity features for the objective function in for KCTP case can be easily found following a similar mathematical procedure to the one presented in the 2-CTP case. It is possible to see that the Hessian matrix of the objective, the first-order principal minor determinant, and the second-order principal minor determinant, have the same structure as the 2-CTP case. As a consequence, it is possible to claim that the degradation process for the K-CTP case follows the same structure as in the 2-CTP one.

According to Lemma 1, based on the reductio ad absurdum method, the above objective function should degraded to

$$
\begin{aligned}
& \text { minimize } Z=\sum_{i=1}^{N} x_{1, j}+\sum_{i=1}^{N} x_{2, j}+\ldots \sum_{i=1}^{N} x_{K, j}
\end{aligned}
$$

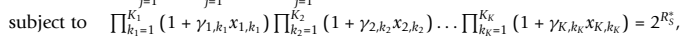


Initialize $\lambda$ according to (8)

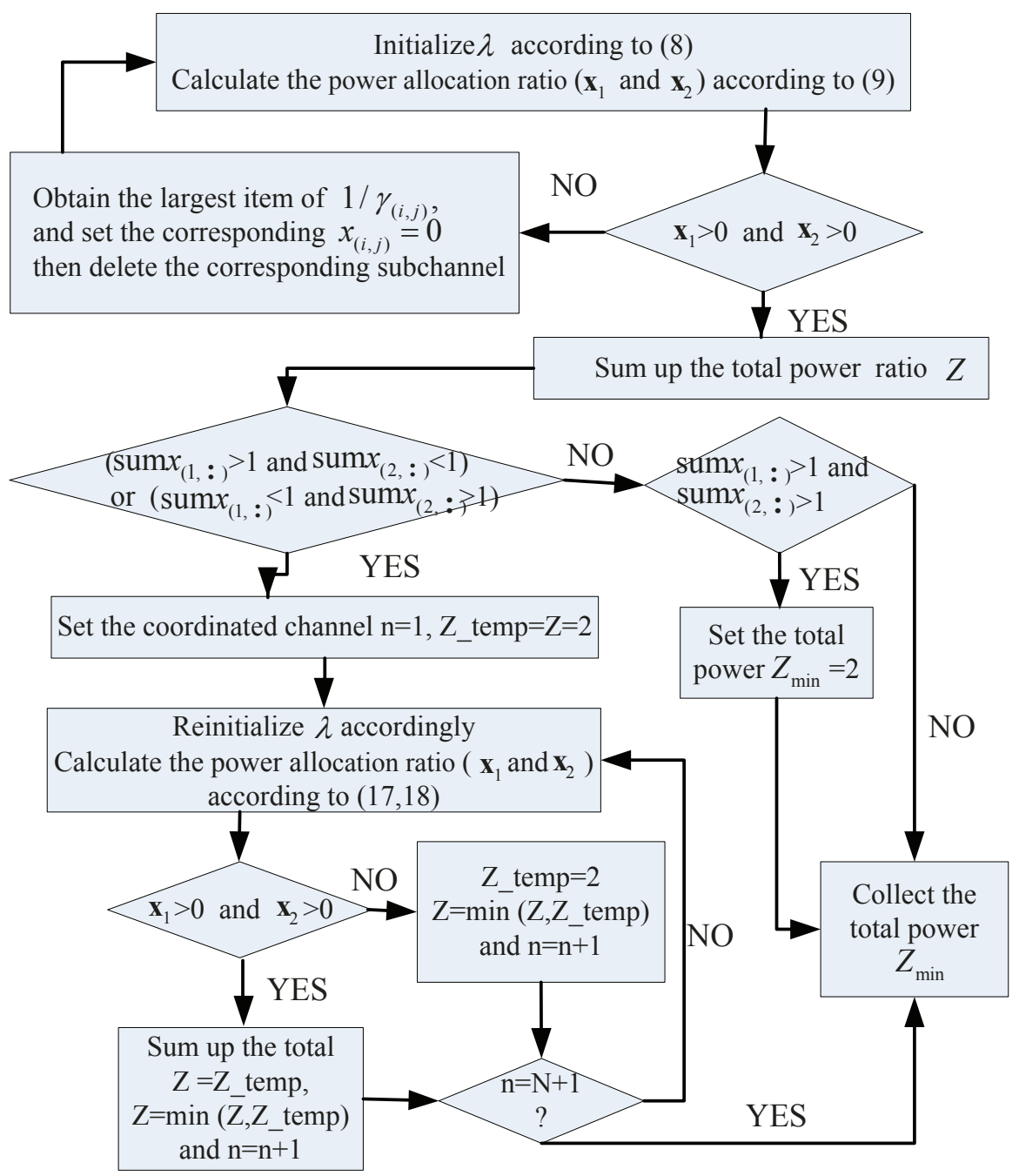

Figure 4 Iterative JMPC-PA power allocation algorithm.

where $\gamma_{i, j}$ for $i \in(1, \ldots K)$ is biggest in $A_{i}$, with $\sum_{i=1}^{K} K_{i}=N$. The degradation implies that $K_{1}$ subchannels should be allocated power by CTP $1, K_{2}$ subchannels by CTP 2, and so on.

By Lagrange dual function, we have that

$$
\begin{aligned}
\Gamma & =\sum_{j=1}^{N} x_{1, j}+\sum_{j=1}^{N} x_{2, j}+\ldots \sum_{j=1}^{N} x_{K, j} \\
& -\lambda\left[\prod_{k_{1}}^{K_{1}}\left(1+\gamma_{1, k_{1}} x_{1, k_{1}}\right) \prod_{k_{2}}^{K_{2}}\left(1+\gamma_{2, k_{2}} x_{2, k_{2}}\right) \ldots \prod_{k_{K}}^{K_{K}}\left(1+\gamma_{K, k_{K}} x_{K, k_{K}}\right)-2^{R_{s}}\right] .
\end{aligned}
$$

First, we derive $\lambda$ as

$$
\lambda=\frac{1}{2^{R_{S}^{*}}}\left(\frac{2^{R_{S}^{*}}}{\prod_{k_{1}=1}^{K_{1}} \gamma_{1, k_{1}} \prod_{k_{2}=1}^{K_{2}} \gamma_{2, k_{2}} \ldots \prod_{k_{K}=1}^{K_{K}} \gamma_{K, k_{K}}}\right)^{\frac{1}{N}} .
$$

Then, the closed-form solution of power allocation ratio for each CTP is obtained as follows:

$$
\begin{array}{ll}
x_{1, k_{1}}=\lambda 2^{R_{S}^{*}}-\frac{1}{\gamma_{1, k_{1}}} & k_{1} \in\left\{1, \ldots, K_{1}\right\}, \\
\vdots & \\
x_{K, k_{K}}=\lambda 2^{R_{S}^{*}}-\frac{1}{\gamma_{K, k_{K}}} \quad k_{K} \in\left\{1, \ldots, K_{K}\right\} .
\end{array}
$$

Finally, the minimum total power consumption ratio is given by

$$
Z_{\min }=N\left(\frac{2^{R_{s}^{s}}}{\prod_{k_{1}=1}^{K_{1}} \gamma_{1, k_{1}} \prod_{k_{2}=1}^{K_{2}} \gamma_{2, k_{2}} \cdots \prod_{k_{K}=1}^{K_{K}} \gamma_{K, k_{k}}}\right)^{\frac{1}{N}}-\sum_{k_{1}=1}^{K_{1}} \frac{1}{\gamma_{1, k_{1}}} \cdots-\sum_{k_{k}=1}^{K_{K}} \frac{1}{\gamma_{K, k_{k}}} .
$$




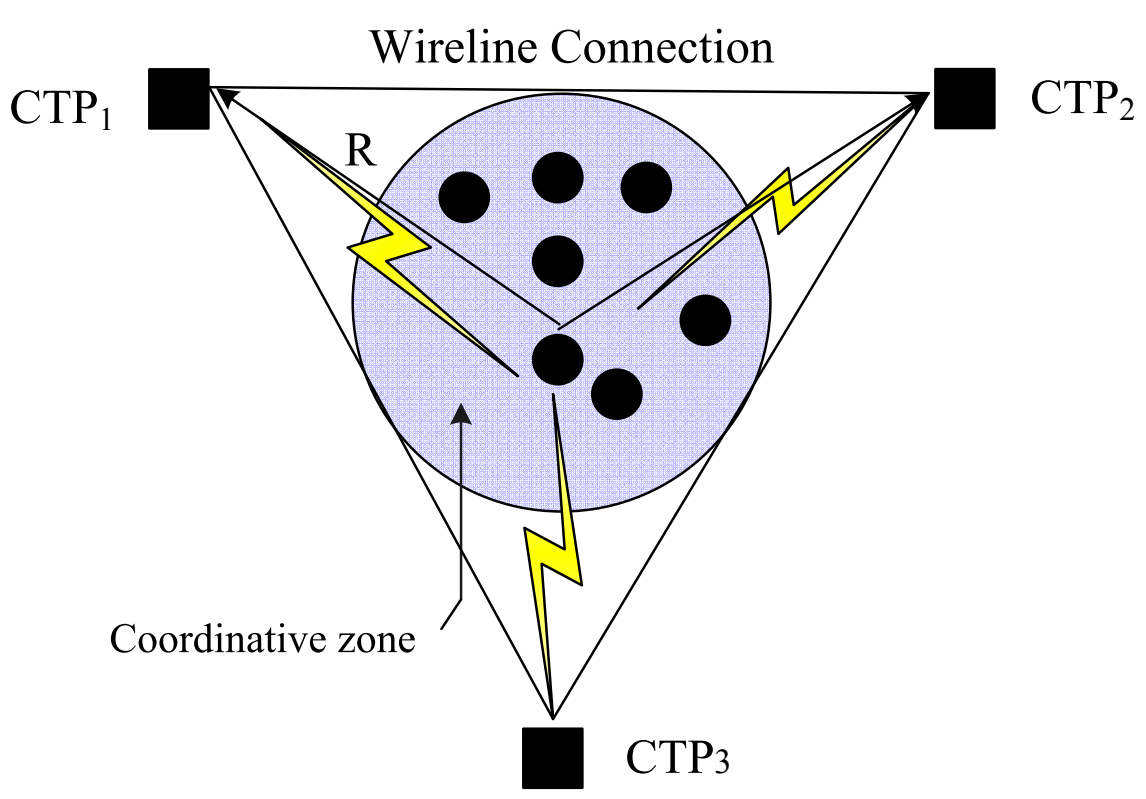

Figure 5 System model with three coordinated transmission points (CTP).

It is indicated that $Z_{\min }$ in (26) is the optimal solution of the primal objective only when the power constraint $\sum_{k_{i}=1}^{K_{i}} x_{i, k_{i}} \leq 1$ for $i \in\{1, \ldots, K\}$ holds. However, if the user density is uneven distributed, some of the CTPs may exceeds the power constraint, and the solution in (9) leads to $\sum_{k_{i}=1}^{K_{i}} x_{i, k_{i}}>1$ for $i \in\{1, \ldots, K\}$. Therefore, the overloaded CTPs should provide their full power for transmission and, in order to meet the throughput requirement, the power allocation in the other CTPs needs to be recalculated. Without loss of generality, we assume $S$ CTPs exceed their power constraint. In this situation, the problem can be described as

$$
\begin{aligned}
& \text { minimize } Z=\underbrace{1+\cdots+1}_{S}+\sum_{j=1}^{N} x_{S+1, j}+\sum_{j=1}^{N} x_{S+2, j} \ldots+\sum_{j=1}^{N} x_{K, j} \\
& \text { subject to }\left(1+\gamma_{1,1} x_{1,1} \ldots+\gamma_{K, 1} x_{K, 1}\right) \ldots\left(1+\gamma_{1, N} x_{1, N} \ldots+\gamma_{K, N} x_{K, N}\right)=2^{R_{S}^{*}},
\end{aligned}
$$

where $\sum_{j=1}^{N} x_{i, j}=1$ for $i \in\{1, \ldots S\}$.

By Lagrange dual function we have

$$
\begin{aligned}
\Gamma & =\underbrace{1+\cdots+1}_{S}+\sum_{j=1}^{N} x_{S+1, j} \cdots+\sum_{j=1}^{N} x_{K, j}-\lambda\left\{\left(1+\gamma_{1,1} x_{1,1} \cdots+\gamma_{K, 1} x_{K, 1}\right) \cdots\right. \\
& {\left[1+\gamma_{1, N}\left(1-x_{1,1}-\cdots-x_{1, N-1}\right) \cdots+\gamma_{S, N}\left(1-x_{S, 1}-\cdots-x_{S, N-1}\right)\right.} \\
& \left.\left.+\gamma_{S+1, N} x_{S+1, N} \cdots+\gamma_{K, N} x_{K, N}\right]-2^{R_{S}^{*}}\right\}
\end{aligned}
$$

For the sake of mathematic simplification, we also denote $A_{j}=\left(1+\gamma_{1,1} x_{1,1} \ldots+\gamma_{K, 1} x_{K, 1}\right)$ and obtain the Hessian matrix $\nabla^{2} f$ as follows

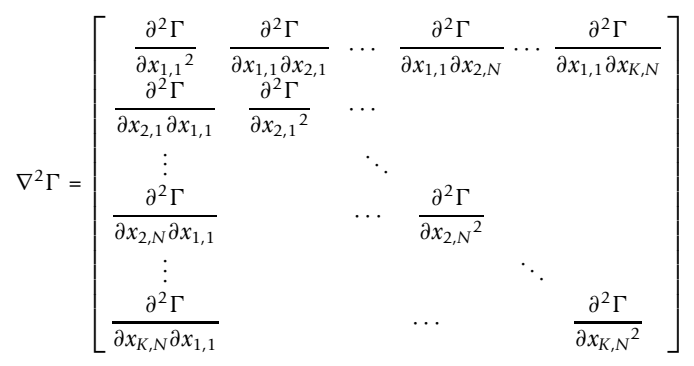

The first-order principal minor determinant and the second-order principal minor determinant have the same structure as the 2-CTP case, which means the Hessian $\nabla^{2} \Gamma$ is indefinite since both the first and second-order principal minor determinant are negative. Therefore, the minimum value must be achieved on the domain boundary, and the degradation process also follows the 2-CTP case.

According to Lemma 2, it is known that only one of the $N$-subchannel should be transmitted together by $S+1$ CTPs (the added one has the biggest $\gamma_{S+t, n}$ for $t \in(S+$ $1, \ldots, K)$ in $A_{n}$ ); the others, according to their different channel gains, should be separated into $K$ parts and transmitted by single CTP Without loss of generality, we assume the $n$th subchannel is the common subchannel. Then, a group of $K_{1}$ subchannels is selected to be used exclusively by CTP 1 , another group of $K_{2}$ subchannels is selected to be used solely by CTP 2 , and so on (i.e., until 


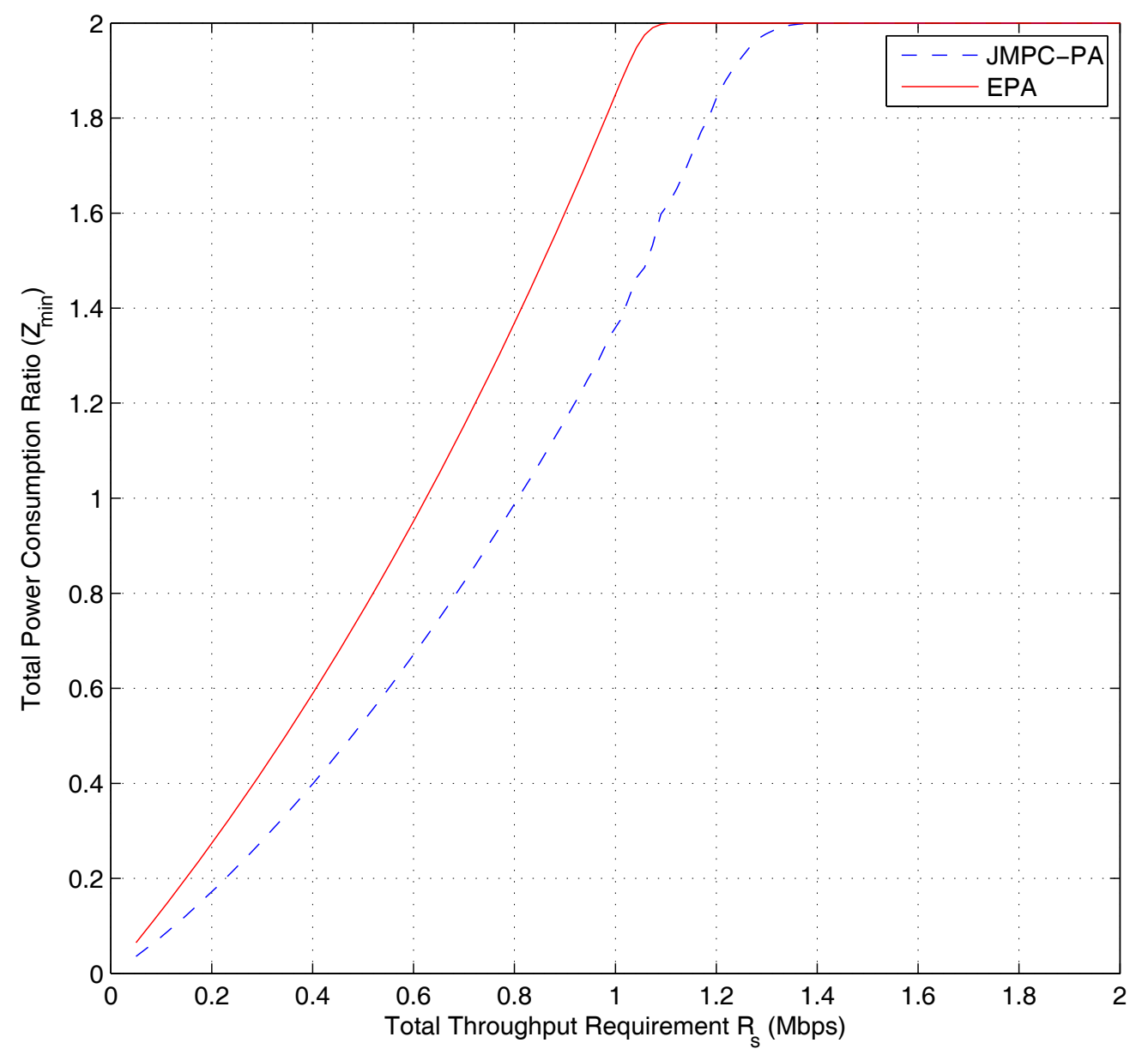

Figure 6 Total power consumption ratio versus total throughput requirement $R_{S}$ (Mbps), 2-CTP case.

the $K$ CTPs receive their own set of channels). Then, the objective function (27) can be expressed as

$$
\begin{aligned}
\Gamma= & \underbrace{1+\cdots+1}_{S}+\sum_{j=1}^{N} x_{S+1, j} \cdots+\sum_{j=1}^{N} x_{K, j}-\lambda\left[\prod_{k_{1}}^{K_{1}}\left(1+\gamma_{1, k_{1}} x_{1, k_{1}}\right) \ldots\right. \\
& \left.\prod_{k_{K}}^{K_{K}}\left(1+\gamma_{K, k_{K}} x_{K, k_{K}}\right)\left(1+\gamma_{1, n} x_{1, n} \ldots+\gamma_{S, n} x_{S, n}+\gamma_{S+t, n} x_{S+t, n}\right)-2^{R_{S}^{*}}\right]
\end{aligned}
$$

where $\gamma_{S+t, n}$ for $t \in(S+1, \ldots K)$ is biggest in $A_{n}$, and $\sum_{k_{i}=1}^{K_{i}} x_{i, k_{i}}+x_{i, n}=1$ for $i \in\{1, \ldots S\}$ with $K_{1} \cdots+K_{K}+1=$ $N$. By Lagrange dual function, we derive $\lambda$ as

$$
\lambda=\frac{1}{2^{R_{S}^{*}}}\left(\frac{2^{R_{S}^{*}}\left(\frac{\gamma_{1, n}}{\gamma_{S+t, n}}\right)^{K_{1}}\left(\frac{\gamma_{2, n}}{\gamma_{S+t, n}}\right)^{K_{2}} \ldots\left(\frac{\gamma_{S, n}}{\gamma_{S+t, n}}\right)^{K_{S}}}{\gamma_{S+t, n} \prod_{k_{1}=1}^{K_{1}} \gamma_{1, k_{1}} \prod_{k_{2}=1}^{K_{2}} \gamma_{2, k_{2}} \cdots \prod_{k_{K}=1}^{K_{K}} \gamma_{K, k_{K}}}\right)^{\frac{1}{N}} .
$$

Similarly, according to Equations (9) and (18), closedform solutions for K-CTP system can be obtained.

\section{Numerical simulations}

In this section, the performance of 2-CTP and 3-CTP systems are considered, since they represent a more reasonable network topology for a practical system implementation. In order to compare the proposed JMPC-PA scheme with EPA, we consider that scheduling is done in a Round Robin fashion. We assume that 20 independent and identically distributed (i.i.d.) Rayleigh-faded users are uniformly located within the coordinative zone (i.e., the colored zone of Figure 1 and Figure 5). Let us assume that distance $R$ is 1000 meters, while distance No-co $R$ is $600 \mathrm{~m}$. Path loss model is Okumura-Hata: $l(d)=137.74+35.22 \lg (d)$ in $\mathrm{dB}$. Standard deviation for 


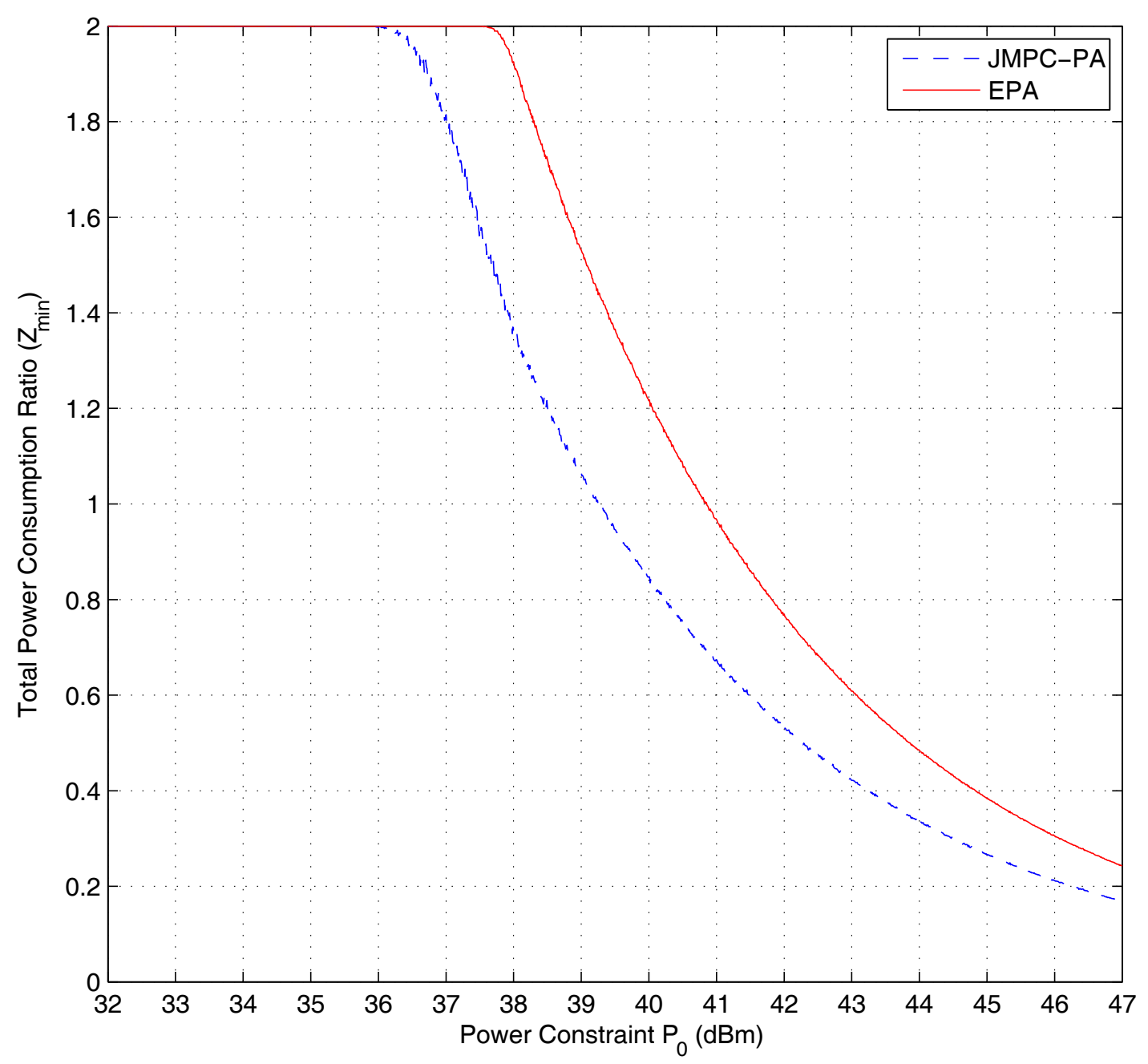

Figure 7 Total power consumption ratio versus power constraint $P_{0}(\mathrm{dBm}), 2$-CTP case.

shadowing is $3.65 \mathrm{~dB}$. For the sake of simplification, we assume that the total bandwidth is $B=1 \mathrm{MHz}$, that the total number of subchannels is $N=25$, and that the noise power in downlink is $\sigma^{2}=-105 \mathrm{dBm}$ in all subcarriers. The total power consumption of each power allocation scheme is averaged over 1000 independent realizations of users' location by Monte-Carlo simulation.

Under different total throughput constraints $R_{S}$ and each CTP's power constraints $P_{0}(\mathrm{dBm})$, the minimum total power consumption of the proposed JMPC-PA scheme and EPA are compared.

(1) 2-CTP case

Given a transmission power constraint $P_{0}=42 \mathrm{dBm}$, Figure 6 indicates that the proposed power allocation scheme consumes less power than EPA under different throughput requirement (e.g., when $R_{s}=1 \mathrm{Mbps}$, EPA needs $Z=1.22$ while JMPC-PA only requires $Z=0.86$ ). Actually, the ability of achieving throughput in EPA is 1.1 Mbps, due to the power constraint at each CTP. However, in our proposed JMPC-PA, the achieving throughput is $1.38 \mathrm{Mbps}$ when both he transmitted power reached the constraint. Figure 7 shows that under a given throughput requirement $R_{s}=0.5 \mathrm{Mbps}$, the proposed scheme consumes less power compared to EPA at both, low power constraint and high power constraint (e.g., when $P_{0}=40 \mathrm{dBm}$, EPA needs $Z=1.86$ while JMPC-PA only requires $Z=1.36$ ). It can be seen that when the power constraint is lower than $36 \mathrm{dBm}$, neither of the two schemes could achieve the throughput requirement 


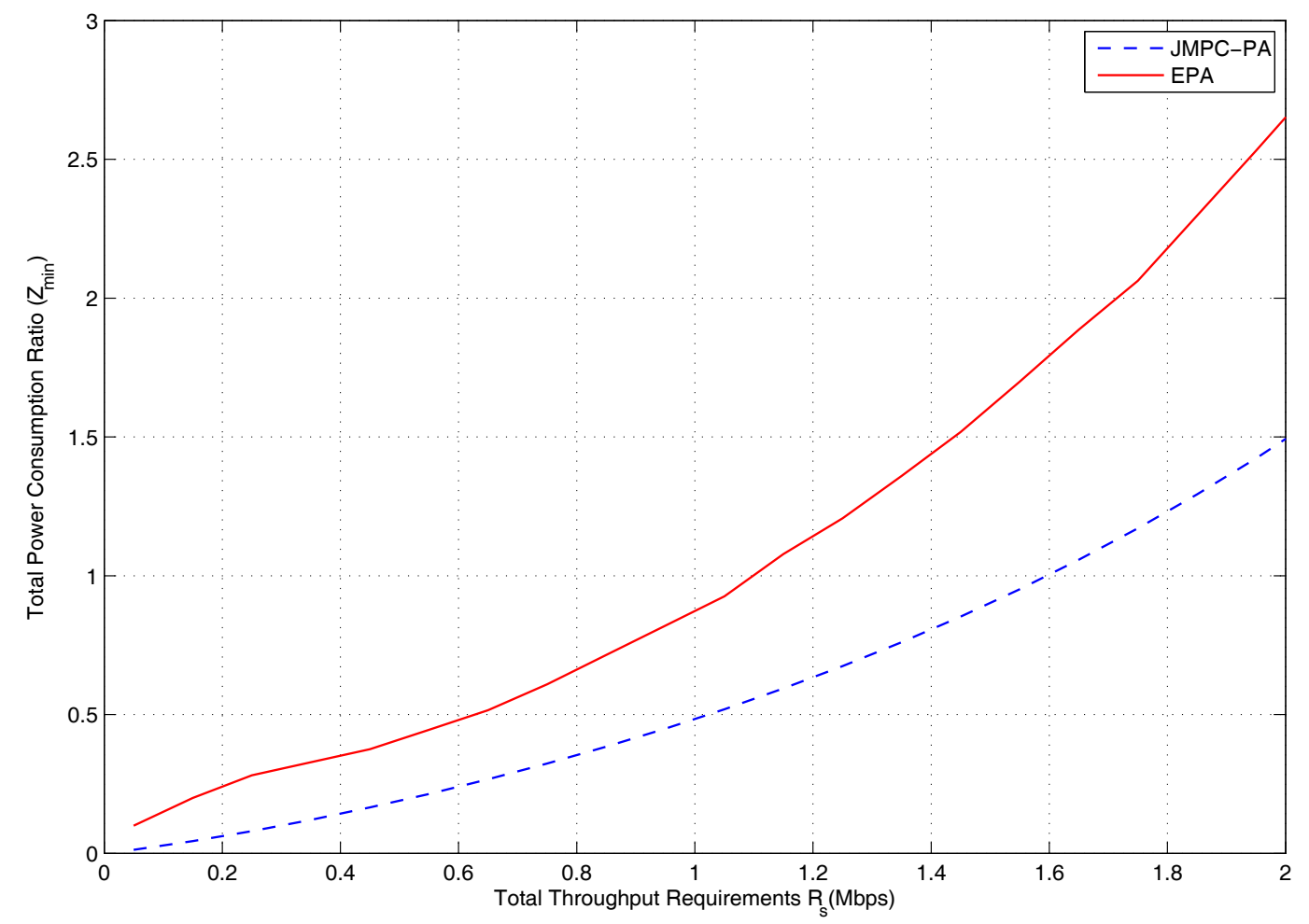

Figure 8 Total power consumption ratio versus total throughput requirement $R_{S}$ (Mbps), 3-CTP case.

$R_{s}=0.5 \mathrm{Mbps}$. Yet, the proposed JMPC-PA can meet the throughput requirement when the power constraint is larger than $36 \mathrm{dBm}$, compared to the $37.5 \mathrm{dBm}$ that are required in the EPA scheme. Actually, when the power constraint is large enough, the "cooperating" transmitting mode will not occur (this is the explanation for the reduction in the gain gap that is observed as the power constraint increases). To sum up it is possible to conclude that, under the perfect coordinated transmission between two CTPs, the proposed JMPC-PA scheme provides an important improvement in the total power consumption of the system.

(2) 3-CTP case

The power constraint of each CTP in the 3-CTP case is equal to the one in the 2 -CTP case plus $10 \log _{10}(2 / 3)$ $\mathrm{dBm}$, so that the total power that is available in the system is identical in both cases (and fair comparisons are possible). As expected, the results in this case are similar the the ones obtained for the 2-CTP situation. Figure 8 indicates the proposed JMPC-PA scheme consumes less power than EPA in presence of different throughput requirements. Similarly, Figure 9 shows that when the throughput requirement is $R_{s}=0.5 \mathrm{Mbps}$, the proposed JMPC-PA scheme consumes less power compared to
EPA in both, low power constraint and high power constraint. However, comparing Figure 8 with Figures 6, 7, and 9, we observe that the performance of the 3-CTP case is better than the performance of the 2-CTP case for both, JMPC-PA scheme and EPA scheme. The reason for this behavior is that in the 3-CTP case, the system has more options to choose the power allocation in each CTP to provide better service.

\section{Concluding remarks}

A novel power allocation scheme for the downlink of an OFDMA system that implements CoMP transmission was investigated in this article. The ultimate goal of the proposed scheme was to minimize the total transmit power consumption, verifying at the same time the target throughput of the system and the individual power constraint per CTP. The closed-form solution for the optimal power allocation was obtained, not only for the simpler 2-CTP case, but also for the more general $K$ CTP case with an arbitrary number of coordinated sources. Based on the derived solutions, a novel power allocation algorithm with JMPC-PA was proposed. Important reductions on the power consumption were observed in different situations, when comparing the 


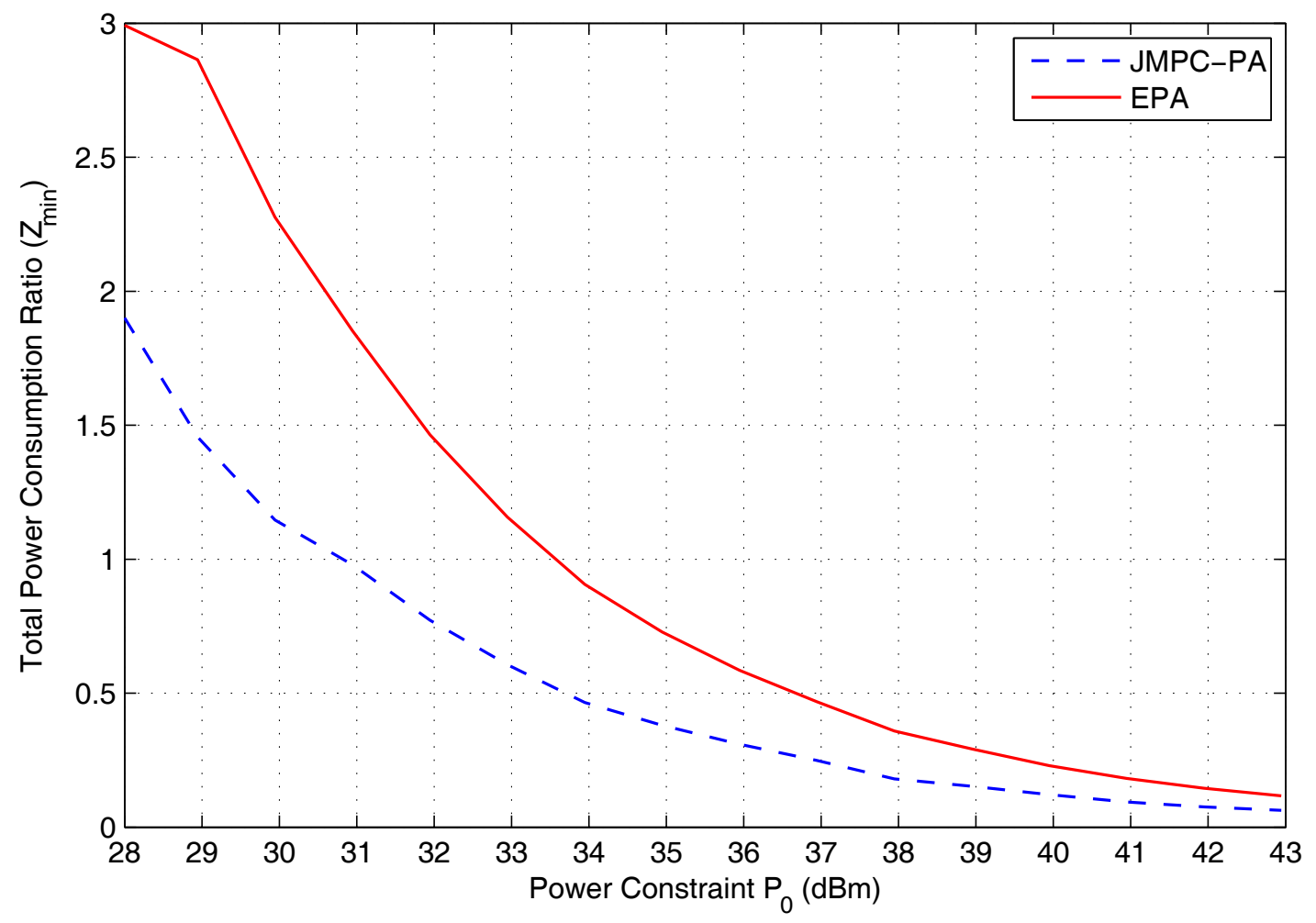

Figure 9 Total power consumption ratio versus power constraint $P_{0}(\mathrm{dBm}), 3-\mathrm{CTP}$ case.

performance of the JMPC-PA scheme with the one provided by the simple EPA approach. These results show the potential gains that exist when implementing joint power allocation in CoMP transmissions, particularly when energy efficiency issues become a main concern.

\section{Endnote}

${ }^{a}$ Note that different scheduling strategies simply induce different channel gain statistics for the selected subcarriers [18].

\section{Acknowledgements}

This study was supported by China-EU International Scientific and Technological Cooperation Program (0902), National Nature Science Foundation of China (Grant No. 61001119) and Fund for Creative Research Groups of China (Grant No. 61121001).

\section{Author details}

'Key Laboratory of Universal Wireless Communications, Ministry of Education, Beijing University of Posts and Telecommunications, Beijing, China ${ }^{2}$ Department of Communications and Networking, Aalto University, Helsinki, Finland ${ }^{3}$ StarPoint Communication Software Company Ltd., Beijing 100102, China

\section{Competing interests}

The authors declare that they have no competing interests.
Received: 30 June 2011 Accepted: 27 March 2012

Published: 27 March 2012

\section{References}

1. H Sampath, S Talwar, J Tellado, V Erceg, A Paulraj, A fourthgeneration MIMO-OFDM broadband wireless system: design, performance, and field trial results. IEEE Commun Mag. 40(9), 143-149 (2002). doi:10.1109/ MCOM.2002.1031841

2. TS Rappaport, A Annamalai, RM Buehrer, WH Tranter, Wireless communications: past events and a future perspective. IEEE Commun Mag. 40(5), 148-161 (2002)

3. AJ Goldsmith, SG Chua, Variable-rate variable-power MQAM for fading channels. IEEE Trans Commun. 45, 1218-1230 (1997). doi:10.1109/26.634685

4. J Jang, KB Lee, Transmit power adaptation for multiuser OFDM systems. IEEE J Sel Areas Commun. 21(2), 171-178 (2003). doi:10.1109/ JSAC.2002.807348

5. Z Shen, JG Andrews, BL Evans, Adaptive resource allocation in multiuser OFDM systems with proportional rate constraints. IEEE Trans Wirel Commun. 4(6), 2726-2737 (2005)

6. W Rhee, JM Cioffi, Increasing in capacity of multiuser OFDM system using dynamic subchannel allocation, in Proc Vehicular Tech Conf 2000 Spring, Tokyo, Japan, (May, 2000)

7. CY Wong, CY Tsui, RS Cheng, KB Letaief, A realtime sub-carrier allocation scheme for multiple access downlink OFDM transmission, in Proc Vehicular Tech Conf 1999 Fall, Amsterdam, Holland, (September, 1999)

8. HS Kim, JS Kwak, JM Choi, JH Lee, Efficient subcarrier and bit allocation algorithm for OFDMA system with adaptive modulation, in Proc Vehicular Tech Conf 2004 Spring, Milan, Italy, (May, 2004)

9. D Kivanc, G Li, H Liu, Computationally efficient bandwidth allocation and power control for OFDMA. IEEE Trans Wirel Commun. 2, 1150-1158 (2003). doi:10.1109/TWC.2003.819016 
10. A Scaglione, DL Goeckel, JN Laneman, Cooperative communications in mobile ad hoc networks. IEEE Signal Process Mag. 23(5), 18-29 (2006)

11. Y Jing, B Hassibi, Distributed space-time coding in wireless relay networks. IEEE Trans Wirel Commun. 5, 3524-3536 (2006)

12. M-k Chang, S-y Lee, Performance analysis of cooperative communication system with hierarchical modulation over rayleigh fading channel. IEEE Trans Wirel Commun. 8(6), 2848-2852 (2009)

13. L Venturino, N Prasad, $X$ Wang, Coordinated scheduling and power allocation in downlink multicell OFDMA networks. IEEE Trans Veh Technol. 58(6), 2835-2848 (2009)

14. 3GPP TR 36.814 V0.4.1. Further Advancements for E-UTRA Physical Layer Aspects, (Feb 2009)

15. B Luo, QM Cui, H Wang, XF Tao, P Zhang, Closed form solutions of joint water-filling for coordinated transmission. IEICE Transmission Commun. E93B(12), 3461-3468 (2010). doi:10.1587/transcom.E93.B.3461

16. JL Troutman, Variational Calculus and Optimal Control: Optimization with Elementary Convexity, 2nd edn. (Springer-Verlag, New York, 1996)

17. S Boyd, L Vandenberghe, Convex Optimization, (Cambridge University Press, Cambridge, UK, 2004)

18. M Andrews, K Kumaran, K Ramanan, A Stoylar, P Whiting, R Vijayakumar, Providing quality of service over a shared wireless link. IEEE Commun Mag. 39(2), 150-154 (2001). doi:10.1109/35.900644

doi:10.1186/1687-1499-2012-122

Cite this article as: Cui et al:: Closed-form solution for minimizing power consumption in coordinated transmissions. EURASIP Journal on Wireless Communications and Networking 2012 2012:122.

\section{Submit your manuscript to a SpringerOpen ${ }^{\mathcal{O}}$ journal and benefit from:}

- Convenient online submission

- Rigorous peer review

- Immediate publication on acceptance

- Open access: articles freely available online

- High visibility within the field

- Retaining the copyright to your article

Submit your next manuscript at $\gg$ springeropen.com 